OPEN ACCESS

Edited by: Duan Chen, Norwegian University of Science and Technology, Norway

Reviewed by:

Rinaldo Pellicano, Molinette Hospital, Italy

Minjun Chen,

National Center for Toxicological Research (FDA), United States

*Correspondence: Einar S. Björnsson einarsb@landspitali.is

Specialty section: This article was submitted to Gastrointestinal and Hepatic Pharmacology, a section of the journal Frontiers in Pharmacology

Received: 23 November 2021 Accepted: 24 January 2022 Published: 10 February 2022

Citation:

Björnsson ES, Vucic V, Stirnimann G and Robles-Díaz M (2022) Role of Corticosteroids in Drug-Induced Liver Injury. A Systematic Review. Front. Pharmacol. 13:820724. doi: 10.3389/fphar.2022.820724

\section{Role of Corticosteroids in Drug-Induced Liver Injury. A Systematic Review}

\author{
Einar S. Björnsson ${ }^{1,2 *}$, Vesna Vucic ${ }^{3}$, Guido Stirnimann ${ }^{4}$ and Mercedes Robles-Díaz ${ }^{5}$ \\ ${ }^{1}$ Faculty of Medicine, University of Iceland, Reykjavik, Iceland, ${ }^{2}$ Department of Gastroenterology, Landspitali University Hospital \\ Reykjavik, Reykjavik, Iceland, ${ }^{3}$ Department of Nutritional Biochemistry and Dietology, Centre of Research Excellence in Nutrition \\ and Metabolism, National Institute for Medical Research, University of Belgrade, Belgrade, Serbia, ${ }^{4}$ Hepatology, Departement for \\ Visceral Surgery and Medicine, University Hospital Inselspital and University of Bern, Bern, Switzerland, ${ }^{5}$ Unidad de Gestión \\ Clínica de Aparato Digestivo, Instituto de Investigación Biomédica de Málaga-IBIMA, Hospital Universitario Virgen de la Victoria, \\ Facultad de Medicina, Universidad de Málaga, Centro de Investigación Biomédica en Red de Enfermedades Hepáticas y \\ Digestivas (CIBERehd), Málaga, Spain
}

Introduction: Apart from cessation of the implicated agent leading to drug-induced liver injury (DILI), there is no standard therapy for DILI. Corticosteroids have been used in DILI, although their efficacy is unclear. Published data showed either beneficial effects or no improvement associated with steroid therapy. The aim of the current study was to perform a systematic review of the role of corticosteroids in the treatment of DILI.

Methods: A search was performed in PubMed, searching for the terms: "corticosteroids" and "drug-induced liver injury". Observation studies were included, but case reports excluded.

Results: A total of 24 papers were retrieved. Most of these were observational studies on the effects of corticosteroids in moderate/severe DILI $(n=8)$, reports on the corticosteroid treatment in patients with drug-induced autoimmune hepatitis (DI-AlH) $(n=5)$, and effects of corticosteroids in drug-induced fulminant acute liver failure (ALF, $n=2)$. Furthermore, treatment of corticosteroids in patients with liver injury due to check point inhibitors (CPIs) was addressed in nine studies. In moderate/severe DILI, six out of eight studies suggested steroid treatment to be beneficial, whereas two studies showed negative results. All five observational studies on the effects of corticosteroids in DI-AlH showed good therapeutic response with rapid and long lasting effects after discontinuation of corticosteroids and without evidence of relapse. Steroid therapy was not associated with improved overall survival in patients with drug-induced fulminant ALF. CPIs-induced liver injury was found to improve spontaneously in 33-50\% without corticosteroids, and the rate of patients who were treated responded to steroids in 33-100\% (mean 72\%).

Conclusions: The majority of studies analyzing the effects of corticosteroids in moderate/ severe DILI have demonstrated beneficial effects. However, this was not the case in druginduced fulminant ALF. Patients with DI-AlH had an excellent response to corticosteroids. The majority of those with CPIs-induced liver injury responded to corticosteroids; however, patients without treatment usually recovered spontaneously. The observational design and comparison with historical controls in these studies makes it very difficult to draw 
conclusions on the efficacy of corticosteroids in DILI. Therefore, there is a strong need for a randomized controlled trial to properly assess the role of corticosteroids in DILI.

Keywords: DILI, corticosteroids, treatment of DILI, acute liver failure, drug-induced autoimmune hepatitis, druginduced liver injury, check point inhibitors

\section{INTRODUCTION}

Corticosteroids or steroids have been used for several decades in the treatment of autoimmune hepatitis (AIH). Their use is based on placebo-controlled trials dating back to the 1970s. In an early clinical trial including patients with chronic active HBsAgnegative hepatitis, treatment consisted of prednisolone or placebo. In this early trial, 5-years survival was $32 \%$ in patients on placebo but $82 \%$ in steroid treated patients (Kirk et al., 1980). Many patients with suspected drug-induced liver injury (DILI) have histological features that are similar to those observed in AIH (Suzuki et al., 2011; Germani et al., 2021). It is therefore conceivable that patient with DILI might benefit from steroid treatment as well, but data on corticosteroid treatment in DILI is scarce. Apart from AIH, alcoholic hepatitis and acute liver graft rejection in transplanted patients, there are no well documented indications for steroid therapy in patients with liver disease.

\section{MATERIAL AND METHODS}

A systematic review of the published literature was performed with a search of https://pubmed.ncbi.nlm.nih.gov/(via MEDLINE up to October 2021). This systematic review was performed following the PRISMA guidelines. The search was conducted with the following main terms: "Corticosteroids", combined with "drug-induced liver injury". All types of design of clinical human studies with the administration of corticosteroids in DILI were analyzed, provided that the etiology of liver disease was either conventional drug-induced or related to herbal and dietary supplements (HDS).

Included were articles in English assessing the effects of corticosteroids in the treatment of patients diagnosed with DILI due to conventional drugs and HDS; Exclusion criteria were review articles, animal studies and/or basic research studies and liver injury due to other causes than DILI. Furthermore, case reports describing effects of corticosteroids in DILI were excluded.

All publications retrieved in the search were screened for eligibility by different authors (ESB, VV, GS and MR) based on the predetermined criteria listed above. Discrepancies were resolved by majority opinion. The work was supervised by a senior investigator (ESB).

The following data were extracted from the included publications: surname of the first author, year of publication, number of patients, design of the study, type and severity of DILI, doses of corticosteroids given, the duration of therapy and treatment response in terms of numerical values. In patients with acute fulminant liver failure, survival was considered as the treatment response in contrast with the other studies, where normalization of liver tests were considered treatment response, lack of relapse of hepatitis after stopping corticosteroids and no need for additional immunosuppressive drugs.

\section{RESULTS}

A total of 661 publications were retrieved from the database search. Of them, 643 publications were not eligible for the following reasons: being review papers, case reports, animal studies, in other languages than English or being otherwise irrelevant for the aim of the study (Figure 1). After reviewing the references of the included studies and reviews identified in the literature search, six additional publications were retrieved.

Thus, the search revealed a total of 24 studies that fulfilled the predetermined criteria and were analyzed. Most of these were observational studies and clinical studies on the effects of corticosteroids in moderate and severe DILI $(n=8)$, reports on the corticosteroid treatment in patients with drug-induced autoimmune hepatitis (DI-AIH) $(n=5)$, and effects of corticosteroids in drug-induced fulminant acute liver failure (n $=2$ ). Furthermore, treatment of corticosteroids in patients with liver injury due to check point inhibitors was addressed in nine studies. In moderate/severe DILI, six out of eight studies suggested steroid treatment to be beneficial, whereas two studies showed negative results. All five observational studies in DI-AIH showed good therapeutic effects of corticosteroids. Steroid therapy was not associated with improved overall survival or improved survival in patients with drug-induced fulminant acute liver failure. Patients with check point inhibitors-induced liver injury were found to improve spontaneously in $33-50 \%$ without corticosteroids, but most of those who were treated responded to steroid therapy.

\section{Steroid Use in Patients With Moderate and Severe DILI}

Ferrero et al. treated five patients with imatinib induced liver injury with corticosteroids with an excellent response but unfortunately without a control group (2006).

Controlled clinical studies investigating corticosteroid treatment in severe DILI are largely lacking and no placebocontrolled study has been undertaken to date. In a report by Wree et al. (2011) on 15 patients with DILI, nine patients were treated with steroid step-down therapy for several weeks, whereas six patients were treated with a steroid pulse therapy for 3 days (Table 1). Ursodeoxycholic acid (UDCA) was used in all patients. Steroids were given in high doses 


\section{Search in Pubmed for "corticosteroids" AND "drug-induced liver injury"}

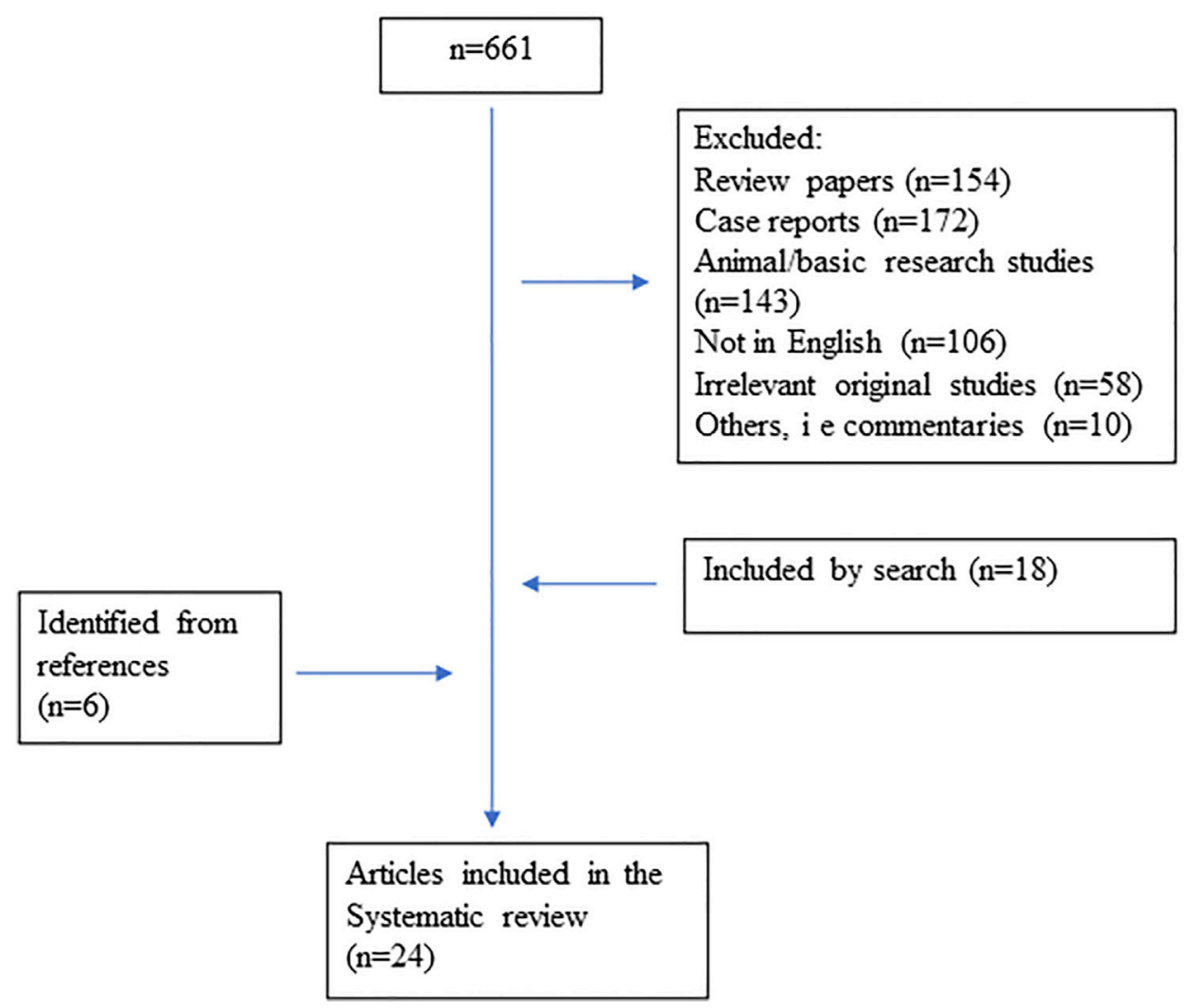

FIGURE 1 | Flow chart over the literature review process.

(2-20 $\mathrm{mg} / \mathrm{kg} /$ day) and patients who received steroids had a decrease in bilirubin and transaminases to less than $50 \%$ of peak value in 2 weeks with normalization all within 8 weeks (Wree et al., 2011). The authors compared the decrease of peak bilirubin level by $50 \%$ after detection of DILI with a report from the DILIN cohort among 220 patients with DILI (Chalasani et al., 2008). In the study by Wree et al. (2011) the median duration from peak bilirubin value to $50 \%$ reduction was 8.5 days, which was significantly $(p<0.05)$ shorter than in the DILIN cohort (Chalasani et al., 2008) which had a median of 13 days. Patients without histological signs of pre-existing liver damage were found to have the most favourable clinical course (Wree et al., 2011). Interestingly, five of these patients developed DILI due to use of anabolic steroids, two as a consequence of phenprocuomon treatment, and one each because of treatment with desflurane, ovarian stimulation for in vitro fertilisation, flutamide, occupational solvent inhalation, trimethoprimsulfamethoxazole, propofol, minocycline and nitrofurantoin (Wree et al., 2011). The last two mentioned drugs, nitrofurantoin and minocycline, have been associated with drug-induced autoimmune hepatitis that often require corticosteroids for improvement. As the control group consisted of historical controls with other drugs leading to DILI, the efficacy of steroids in severe DILI is not clear.

Researchers from Beijing reported beneficial effects of steroids in patients with DILI, too. Among 70 patients with high bilirubin (TBIL $\geq 10 \times$ ULN), 20 were treated with steroid step-down therapy (79 \pm 26 days) and others with non-steroid therapy (Hou et al., 2012). The steroid therapy group showed a higher DILI resolution rate $(p=0.029)$ and a shorter recovery time ( $p=$ 0.012) (Hou et al., 2012) (Table 1).

In a study from Shanghai with 203 DILI patients of whom 53 received steroids but other than that the same management than the non-steroid group (Hu et al., 2016), corticosteroid therapy was associated with improvement in recovery rate from 77 to $88 \%$ in the higher $50 \%$ quartile of total bilirubin (TB) values (Table 1). Administration of corticosteroids was found to hasten the resolution of liver injury by shortening the reduction of TB to $50 \%$ of peak value from 17 to 12 days $(p<0.05)$. The interpretation by the authors was that corticosteroids were beneficial in patients with severe DILI (TB > $243 \mu \mathrm{mol} / \mathrm{L}$ ) who might be at risk of developing ALF (Hu et al., 2016).

However, Pang et al. (2018) reported the results of thirty-two acute DILI patients who received steroid therapy. In these patients no improvement in the recovery time of ALT or 
TABLE 1 | Studies evaluating corticosteroids in different types of DILI are illustrated. The design of the study, the proportion treated with corticosteroids, the control group, doses and median duration of corticosteroids and the overall effects are shown.

\begin{tabular}{|c|c|c|c|c|c|c|}
\hline & $\begin{array}{l}\text { Type of } \\
\text { DILI }\end{array}$ & Design & $\begin{array}{l}\text { Number of patients } \\
\text { treated with steroids }\end{array}$ & Control group & $\begin{array}{c}\text { Dose and duration of } \\
\text { therapy }\end{array}$ & Respone \\
\hline Ferrero et al. (2006) & $\begin{array}{l}\text { Imatinib ind; } \\
\text { DILI }\end{array}$ & Observational & $5 / 5(100 \%)$ & None & 20-40 mg, 20-24 weeks & $100 \%$ \\
\hline Wree et al. (2011) & $\begin{array}{l}\text { Mod./severe } \\
\text { DILI }\end{array}$ & $\begin{array}{l}\text { Observational, All were } \\
\text { on UDCA }\end{array}$ & $15 / 15(100 \%)$ & Historical control & $\begin{array}{l}2-5 \mathrm{mg} / \mathrm{kg} \text { or } 15-20 \mathrm{mg} / \mathrm{kg} \mathrm{\#} \\
\text { Several weeks }\end{array}$ & $100 \%$ \\
\hline Hou et al. (2012) & $\begin{array}{l}\text { Mod./severe } \\
\text { DILI }\end{array}$ & $\begin{array}{l}\text { Observational } \\
\text { Patients had jaundice }\end{array}$ & $33 / 300$ (11\%) & $\begin{array}{l}\text { Non-steroid } \\
\text { therapy }\end{array}$ & - & $100 \%$ \\
\hline Hu et al. (2016) & $\begin{array}{l}\text { Mod./severe } \\
\text { DILI }\end{array}$ & $\begin{array}{l}\text { Observational } \\
\text { Patients had jaundice }\end{array}$ & $53 / 203$ (26\%) & $\begin{array}{l}\text { Non-steroid } \\
\text { therapy }\end{array}$ & $\begin{array}{l}40-60 \text { mg or } 60-120 \text { mg, } \\
\text { \{Several weeks }\end{array}$ & $90.6 \%$ \\
\hline Pang et al. (2018) & Severe DILI & $\begin{array}{l}\text { Observational } \\
\text { Severity score } 3\end{array}$ & $32 / 32$ (100\%) & $\begin{array}{l}\text { Non-steroid } \\
\text { therapy }\end{array}$ & - & $94 \%$ \\
\hline Borlak et al. (2018) & Flupirtine & $\begin{array}{l}\text { Observational } \\
\text { All were on NAC }\end{array}$ & $21 / 21(100 \%)$ & Historical controls & $\begin{array}{l}1 \mathrm{mg} / \mathrm{kg} \\
\text { "stopped when ALT } \\
\text { normalized" }\end{array}$ & $90.5 \%$ \\
\hline Wan et al. (2019) & $\begin{array}{l}\text { Mod./severe } \\
\text { DILI }\end{array}$ & Observational & 66/90 (73\%) & $\begin{array}{l}\text { Non-steroid } \\
\text { therapy }\end{array}$ & $\begin{array}{l}40 \mathrm{mg} \\
13 \text { weeks }\end{array}$ & $100 \%$ \\
\hline Weber et al. (2019) & $\begin{array}{l}\text { Mod./severe } \\
\text { DILI }\end{array}$ & Observational & $21 / 21(100 \%)$ & Patients with $\mathrm{AlH}$ & $\begin{array}{l}60 \mathrm{mg} \\
19 \text { weeks }\end{array}$ & $91 \%$ \\
\hline Björnsson et al. (2010) & DI-AlH & Observational & 24/24 (100\%) & Patients with $\mathrm{AlH}$ & 20-40 mg, 8 weeks & $100 \%$ \\
\hline Ghabril et al. (2013) & DI-AlH & Observational & 12/34 (35\%) & No controls & - & $100 \%$ \\
\hline Rodrigues et al. (2015) & DI-AlH & Observational & $8 / 8$ (100\%) & No controls & - & $100 \%$ \\
\hline Björnsson et al., 2017 & DI-AlH & Observational & $9 / 15$ (60\%) & No controls & 20-40 mg, 8 weeks & $100 \%$ \\
\hline Björnsson et al. (2022) & $\begin{array}{l}\text { Infliximab ind. } \\
\text { DILI }\end{array}$ & Observational & 17/36 (47\%) & $\begin{array}{l}\text { Non-steroid } \\
\text { therapy }\end{array}$ & 20-40 mg, 8 weeks & $100 \%$ \\
\hline Rakela et al. (1991) & $\begin{array}{l}\text { ALF due to } \\
\text { drugs }\end{array}$ & Observational & $44 / 62(71 \%)$ & Placebo & $\begin{array}{l}400-800 \mathrm{mg}^{*} \\
-\end{array}$ & $9-24 \%$ \\
\hline Karkhanis et al. (2014) & $\begin{array}{l}\text { ALF due to } \\
\text { drugs }\end{array}$ & Observational & 25/66 (38\%) & $\begin{array}{l}\text { Non-steroid } \\
\text { therapy }\end{array}$ & $\begin{array}{l}42 \mathrm{mg} \\
5 \text { weeks }\end{array}$ & $61 \%$ \\
\hline Huffman et al. (2018) & $\begin{array}{l}\text { DIILI due to } \\
\text { CPIs }\end{array}$ & Observational & 16/17 (94\%) & No controls & $\begin{array}{l}1 \mathrm{mg} / \mathrm{kg} / \text { day } \\
6 \text { weeks }\end{array}$ & $81 \%$ \\
\hline De Martin et al. (2018) & $\begin{array}{l}\text { DILI due to } \\
\text { CPIs }\end{array}$ & Observational & $10 / 16(63 \%)$ & $\begin{array}{l}\text { Non-steroid } \\
\text { therapy (37\%) }\end{array}$ & 1 mg/kg/day & $90 \%$ \\
\hline Cheung et al., 2019 & $\begin{array}{l}\text { DILI due to } \\
\text { CPIs }\end{array}$ & Observational & 19/21 (90.5\%) & No controls & $\begin{array}{l}1 \mathrm{mg} / \mathrm{kg} / \text { day } \\
50-60 \mathrm{mg}\end{array}$ & $53 \%$ \\
\hline Gauci et al. (2018) & $\begin{array}{l}\text { DILI due to } \\
\text { CPIs }\end{array}$ & Observational & $5 / 10(50 \%)$ & $\begin{array}{l}\text { Non-steroid } \\
\text { therapy }\end{array}$ & $\begin{array}{l}1 \mathrm{mg} / \mathrm{kg} / \text { day } \\
7 \text { weeks }\end{array}$ & $100 \%$ \\
\hline $\begin{array}{l}\text { Riveiro-Barciela et al. } \\
\text { (2020) }\end{array}$ & $\begin{array}{l}\text { DILI due to } \\
\text { CPIs }\end{array}$ & Observational & 28/28 (100\%) & Patients with $\mathrm{AlH}$ & $\begin{array}{l}60 \mathrm{mg} \\
9 \text { weeks }\end{array}$ & $64 \%$ \\
\hline Miller et al. (2020) & $\begin{array}{l}\text { DILI due to } \\
\text { CPIs }\end{array}$ & Observational & $67 / 100$ (67\%) & $\begin{array}{l}\text { Non-steroid } \\
\text { therapy (33\%) }\end{array}$ & 6 weeks & $86 \%$ \\
\hline Kitagataya et al. (2020) & $\begin{array}{l}\text { DILI due to } \\
\text { CPIs }\end{array}$ & Observational & $4 / 17$ (24\%) & No controls & 1-2 mg/kg/day & $50 \%$ \\
\hline Ito et al. (2021) & $\begin{array}{l}\text { DILI due to } \\
\text { CPIs }\end{array}$ & Observational & $30 / 58$ (52\%) & No controls & $0.5-2.0 \mathrm{mg} / \mathrm{kg} /$ day & $33 \%$ \\
\hline Gauci et al. (2021) & $\begin{array}{l}\text { DILI due to } \\
\text { CPIs }\end{array}$ & Observational & $13 / 21(62 \%)$ & $\begin{array}{l}\text { Non-steroid } \\
\text { therapy (38\%) }\end{array}$ & $\begin{array}{l}1 \mathrm{mg} / \mathrm{kg} / \mathrm{day} \\
7 \text { weeks }\end{array}$ & $92 \%$ \\
\hline
\end{tabular}

DILI, drug-induced liver injury.

UDCA, urso deeoxycholic acid, NAC = N-acetylcystein, ALF, acute liver failure; CPIs, Check Point Inhibitors.

\#9 were treated with a steroid step-down therapy with reduction of the daily dose over several weeks (prednisone, range 2-5 mg/kg/day and weekly reduction); six patients received a steroid pulse-therapy for 3 days (prednisone, range $15-20 \mathrm{mg} / \mathrm{kg} /$ day).

\{ Two methods of drug administration. (1) Corticosteroid step-down therapy with a reduction of the daily dose over several weeks (methylprednisolone 60-120 mg/day or prednisone 40-60 mg/day for 3-5 days, and then prednisone at 20 mg/day, and tapered for 5-10 mg weekly thereafter). (2) Corticosteroid pulse therapy, using methy/prednisolone 60-120 mg/day for 3-5 days.

resolution rate was observed compared with the non-steroid group (with otherwise no difference in their management). Thus, the authors concluded that steroid therapy was not associated with an improved recovery time or survival in acute severe DILI patients (Pang et al., 2018) (Table 1).

Borlak et al. from Germany (2018) recently analyzed in a retrospective cohort of patients with flupirtine induced liver injury the effects of oral prednisolone in addition to $\mathrm{N}$-acetylcystein (NAC) (Table 1). Overall, 21 patients with liver injury associated with flupirtine received $\mathrm{NAC} /$ prednisolone and outcome in these patients was compared with an external cohort of 30 cases of flupirtine induced liver injury not receiving this treatment or another therapy (Borlak et al., 2018). The combined treatment was well tolerated and was 
associated with significant ALT, AST and INR improvement within 2 weeks, but patients with jaundice resolved slowly. However, two patients who were said to have started medical treatment late in the course of the liver injury, developed hepatic encephalopathy and required liver transplantation. Normalization in liver tests occurred more rapidly compared with the historical controls including a case with fatal outcome (Borlak et al., 2018). Additional in vitro studies revealed glutathione depletion as an important contribution to the development of DILI (Borlak et al., 2018). As the authors pointed out, prospective randomized clinical trials are needed to confirm efficacy of this treatment in severe DILI.

However, a recent study from China could not reproduce these results (Wan et al., 2019) (Table 1). Overall, 66 out of 90 DILI patients enrolled in this study were treated with steroids, while 24 composed the control group without other medical therapy. Prednisone therapy was not beneficial at a median daily dose of $40 \mathrm{mg}$ for the treatment of severe DILI. When the prednisone group was divided into two subgroups, the high dose group ( $>40 \mathrm{mg}$ per day) was associated with lower rates of severity reduction than the control group or the low dose group $(<40 \mathrm{mg}$ per day) (Wan et al., 2019).

In a recent study from Germany, patients suspected of DILI with acute liver injury (ALI) who were referred to a single centre were assessed in terms of ALT response to corticosteroids (Weber et al., 2019) (Table 1). Among 44 patients with ALI who took at least one drug and who were treated with steroids, 22 had the final diagnosis of AIH and 22 were considered to have DILI. Scores of $\mathrm{AIH}$ and for DILI were calculated at baseline. The decrease in ALT levels was significantly more marked in the patients with the final diagnosis of DILI than in the AIH group. This is somewhat surprising as AIH patients usually respond very well to steroids. However, among the causative agents, minocycline and pembrolizumab are well documented to induce drug induced $\mathrm{AIH}$ (DI-AIH). It is also conceivable that there was some selection among those with a suspicion of DILI who received steroids i.e., 44/288 (15\%) of all patients referred to the study on idiosyncratic DILI. For example, $95 \%$ of the DILI patients treated with steroids had hepatocellular type of injury (Weber et al., 2019).

Thus, is it difficult to draw firm conclusions on the beneficial effects of steroids in DILI in general, but the results are intriguing.

\section{Steroids in Drug-Induced Autoimmune Hepatitis}

Steroids have generally been reported to be beneficial in patients with drug-induced autoimmune hepatitis (Björnsson et al., 2010; Ghabril et al., 2013; Björnsson et al., 2015; Rodrigues et al., 2015; Björnsson et al., 2017; Björnsson et al., 2022) (Table 1). In a study from the DILIN study group in the US, six patients with TNF-alpha inhibitors induced liver injury and additional 28 patients from the literature were analyzed: infliximab $(\mathrm{n}=26)$, etanercept $(\mathrm{n}=4)$ and adalimumab $(n=4)$ (Ghabril et al., 2013). A total of 22 patients had autoimmune features and 12 patients (55\%) received corticosteroid therapy and responded well to therapy. In a study from Portugal with eight patients with TNF-alpha inhibitors induced liver injury (infliximab $=7$, adalimumab $=1$ ), all were treated with corticosteroids and had normalization of liver tests and those who discontinued immunosuppression did not have a relapse of AIH (Rodrigues et al., 2015).

At the current time there is no consensus on the definition of DI-AIH and most studies have presented patients with DIAIH with a clinical diagnosis of DI-AIH with positive autoantibodies and/or IgG (Björnsson et al., 2010; Ghabril et al., 2013; Rodrigues et al., 2015; Björnsson et al., 2022). However, in one study DI-AIH was defined as a drug reaction with well-documented cause of DI-AIH, with positive ANA or SMA or elevated IgG, 10 times upper limit of normal ALT, with hepatocellular (HC) or mixed pattern or requirement of corticosteroids in patients with hepatocellular type, defined as no improvement in ALT and AST after discontinuation of the implicated agent (Björnsson et al., 2017).

Among patients from a single center study of 36 patients with infliximab induced liver injury, $17(47 \%)$ were treated with corticosteroids (Björnsson et al., 2022). Treatment response was good with prompt resolution of liver test abnormalities and liver enzymes normalized in all patients, which was faster than in those who did not receive steroids (Björnsson et al., 2022). The relapse of liver injury was not observed after tapering of corticosteroids despite prolonged follow-up and no patients developed DILI due to a second biologic.

Patients with DI-AIH induced by drugs with well documented ability to induce AIH such as minocycline, hydralazine, methyl-dopa, nitrofurantoin and infliximab, mimic regular AIH clinically, biochemically and immunologically. Thus, as autoimmune features cannot distinguish reliably between DILI and genuine AIH in these patients, steroid treatment is recommended according to clinical guidelines (European Association for the Study of the Liver, 2015).

\section{Steroid Use in Acute Liver Failure}

Drugs are a common cause of acute liver failure (ALF) (Ostapowicz et al., 2002; Björnsson et al., 2005; Wei et al., 2007; Reuben et al., 2010). Some of the patients with ALF have prominent inflammatory features and more than 3 decades ago steroids were initially investigated in ALF (Ware et al., 1974; European Association for the Study of the Liver, 1979; Rakela et al., 1991). These trials failed to show a benefit of steroid treatment compared with placebo. In a randomized, unblinded clinical trial carried out in 17 European centres, ALF developed mostly due to viral hepatitis or presumed viral hepatitis. Patients were given $400 \mathrm{mg}$ hydrocortisone twice a day or no medical therapy (placebo was not used). Only two patients had drug-induced ALF, both in the control group (European Association for the Study of the Liver, 1979). No significant difference in survival between the two groups was achieved. Another steroid trial in ALF from the US included 62 patients with fulminant drug-induced hepatotoxicity (Rakela et al., 1991) (Table 1). Patients received placebo, $400 \mathrm{mg}$ hydrocortisone, or $800 \mathrm{mg}$ 
hydrocortisone, and had a survival rate of $22 \%, 9 \%$ and $24 \%$, respectively. Based on this study, the European Association for the Study of the Liver recommended discontinuing steroid treatment in patients with ALF.

Auto-antibodies are frequent in patients with ALF. Bernal et al. found autoantibodies in $32 \%$ of ALF patients (Bernal et al., 2007). While autoantibodies were absent in patients with ALF induced by paracetamol, they could be detected in $43 \%$ of nonparacetamol ALF patients. AIH scores classified $50 \%$ of cryptogenic cases as 'probable autoimmune hepatitis' (Bernal et al., 2007).

Analysis undertaken by the investigators of the Acute Liver Failure Study Group (ALFSG) suggested that many patients with ALF of indeterminate etiology had histological features indicating an autoimmune pathogenesis (Stravitz et al., 2011). This motivated members of the ALFSG to conduct a retrospective analysis of ALF patients from 1998-2007 (Karkhanis et al., 2014) (Table 1). Overall, 361 patients with ALF were divided into three subgroups based on origin of liver disease: autoimmune, DILI and indeterminate ALF. In the group consisting of 131 patients with ALF due to DILI, only 16 were treated with steroids and 115 received no steroids. Among those classified as autoimmune ALF, 25/66 (38\%) were treated with steroids and of those with ALF of indeterminate etiology 21/164 (13\%) (Karkhanis et al., 2014). Steroid therapy was not associated with improved overall survival or improved survival in any of these categories. In those patients with highest Model for End stage Liver disease (MELD) scores, steroid treatment was even associated with a worse outcome. Steroid therapy was associated with a marginal benefit in spontaneous overall survival ( $35 \%$ vs. $23 \%, p=0.047$ ), but this benefit did not persist in a multivariate analysis (Karkhanis et al., 2014). As the authors pointed out, it is conceivable that the lack of benefit of steroid use might be due to selection bias. Thus, patients who received steroids might have been more ill. However, MELD scores were similar in the two groups and the international normalized ratio of prothrombin time (INR) was actually higher in those who did not receive steroids. Only patients with alanine aminotransferase (ALT) in the higher $50 \%$ of values who received steroids had improved spontaneous survival (54\% in patients with steroid treatment vs $25 \%$ in patients who did not receive steroids, $p=0.003$ ). The authors postulated that higher ALT might be a surrogate marker for an inflammatory process that might be sensitive to corticosteroid therapy (Karkhanis et al., 2014).

\section{Steroid Use in Check Point Inhibitor Induced Liver Injury}

In a recent review, a third type of DILI was introduced, named the indirect type (Hoofnagle and Bjornsson, 2019). This type of DILI is different from direct DILI (such as paracetamol-induced liver toxicity) and idiosyncratic DILI as it has to do with the action of the drug i. e. what the drug does and not what it is. In other words, the action of the drug can affect the balance in the immune system and therefore induce various immune mediated adverse effects. Most commonly implicated agents in indirect DILI are antineoplastic agents such as Check Point Inhibitors (CPI) and other monoclonal antibodies such as infliximab (Björnsson et al., 2022). In a study of 17 melanoma patients with DILI due to CPIs, other concomitant immune-mediated adverse effects were observed in $47 \%$ of patients (gastrointestinal, dermatological, endocrine, lung disorders) (Huffman et al., 2018). Similar results have been reported from larger cohorts (Miller et al., 2020). The frequency of ALT elevations in clinical trials has been found to be 3-15\%, some of them of transient nature, and an increase of 5-20xULN has been observed in up to $3 \%$ of patients (Shah et al., 2020).

In the study of Huffman et al. (2018), 17 patients had any kind of hepatotoxicity (mild, moderate or severe DILI, Table 1). Twelve of 17 were diagnosed after treatment with ipilimumab, three were diagnosed after pembrolizumab, and 2 after ipilimumab combined with nivolumab. Patients were most commonly treated with systemic corticosteroids such as prednisone. Immunosuppression was tapered over a median of 42 days; in three patients steroids had to be reinitiated due to clinical or laboratory worsening of liver enzymes. Normalization of liver tests was seen within a median of 1 month after start of immunosuppression.

In retrospective studies, grade 3-4 ALT elevation were observed in $2-8 \%$ of patients (De Martin et al., 2018; Parlati et al., 2018; Miller et al., 2020). Time to onset varied from 2-24 weeks after initiation of treatment (median 4-16 weeks). Monotherapy with ipilimumab was associated with a higher incidence DILI than other CPIs, but combination of more than one CPI seems to increase the risk of DILI (Shah et al., 2020). DILI due to CPIs has a distinct biochemical and histological phenotype, and often occurs together with other immunemediated adverse reactions. Although considered to be a drug-induced $\mathrm{AIH}$, there are some important clinical and immunological differences between CIP-induced hepatitis and genuine AIH. In most AIH cohorts, $80-90 \%$ of patients are females compared to $50-60 \%$ in CPI induced hepatotoxicity (European Association for the Study of the Liver, 2015; Shah et al., 2020). ANA/SMA is elevated in 80-90\% in AIH but in very few patients with CPI-induced hepatotoxicity (European Association for the Study of the Liver, 2015; Shah et al., 2020).

Clinical guidelines from oncologists recommend high-dose corticosteroids in patients with moderate to severe CPI-induced hepatotoxicity. In grade II hepatotoxicity (ALT 3-5xULN), temporary interruption of CPI therapy is recommended and if liver tests return to baseline within 1-2 weeks, CPI can be resumed (Puzanov et al., 2017; Brahmer et al., 2018; Haanen et al., 2018). In patients with grade III or IV ( $>5 x$ ULN) toxicity, corticosteroids should be given if there is no improvement after discontinuation of therapy and in case of a $>10 x$ ULN elevation permanent interruption is recommended. If the ALT elevation is accompanied by a rise in bilirubin, steroid treatment should be started immediately. Oral prednisone $0.5-1.0 \mathrm{mg} / \mathrm{kg} /$ day and starting iv methylprednisone $1-2 \mathrm{mg}$ per day are recommended in grade 3-4 drug-induced hepatitis (Puzanov et al., 2017; Brahmer et al., 2018; Haanen et al., 2018).

These doses are much higher than those that are used in genuine AIH (European Association for the Study of the Liver, 2015). No clinical trial has been conducted to demonstrate the efficacy of steroids in general and high steroid doses in particular in this patient 
population. Guidline recommendations are mainly empirical and based on recommendations provided in the clinical trial protocols (Puzanov et al., 2017; Brahmer et al., 2018; Haanen et al., 2018).

De Martin et al. (2018) compared the pattern of DILI based on the CPI used, i.e. anti-programmed cell death protein 1(PD-1/PD ligand 1 (PD-L1) and anticytotoxic $\mathrm{T}$ lymphocyte antigen 4 (CTLA-4) monoclonal antibodies (Table 1). Overall, 10/16 patients $(63 \%)$ were treated with corticosteroids, mainly receiving oral corticosteroids, whereas 6 (37\%) improved spontaneously, and in three patients immunotherapy was reintroduced without recurrence of liver injury (De Martin et al., 2018). These results have mostly been reproduced by other groups (Gauci et al., 2018; Miller et al., 2020). In a large cohort from Texas, $33 \%$ of patients, and in a study from Paris, $50 \%$, showed spontaneous improvement in liver tests (Gauci et al., 2018). In two studies, $14-17 \%$ of patients having been treated with steroids exhibited a recurrence of hepatotoxicity after tapering steroids (Pollack et al., 2018; Miller et al., 2020).

Based on the above mentioned studies, 50-67\% of patients with CPI-associated DILI have been treated with steroids, and DILI rarely recurred after steroid tapering (De Martin et al., 2018; Gauci et al., 2018; Parlati et al., 2018; Miller et al., 2020).

In a study from Oxford, 19/21 patients (90.5\%) with hepatotoxicity due to Check Point Inhibitors were treated with corticosteroids (Cheung et al., 2019) (Table 1), but only $50 \%$ had a treatment response, which was similar to a study from Japan (Kitagataya et al., 2020). The study by Ito et al. from Japan was somewhat of an outlier showing a response to steroid treatment in only $33 \%$ of cases (Ito $t$ al. 2021) as shown in Table 1.

In a recent large retrospective cohort study from France consisting of 339 patients with advanced melanoma grade $\geq 3$, hepatotoxicity was observed in 21 patients (Gauci et al., 2021). Thirteen patients were treated with steroids (steroid group, SG), whereas eight were not (non-steroid group, NSG). The median time for resolution of abnormal liver test was 49 days in the SG and 24 days in the NSG ( $p=N S)$. Only one patient had an unfavourable outcome. Two-year survival was $56 \%$ in the SG and $54 \%$ in the NSG ( $\mathrm{p}=\mathrm{NS})$. Higher aminotransferases and bilirubin levels and lower prothrombin levels were observed in the SG than in the NSG (Gauci et al., 2021). The authors concluded that many patients with moderate to severe immune mediated hepatitis due to CPIs can be managed without steroids and suggested that steroid therapy should be considered in patients with high bilirubin and prothrombin values. A management protocol was proposed for validation in large, prospective cohorts (Gauci et al., 2021).

\section{Adverse Effects of Corticosteroid Treatment in Patients With DILI}

There is little data published on adverse effects of high-dose corticosteroid treatment in patients with DILI. Generally, corticosteroid use can be associated with undesirable side effects, such as diabetes mellitus (DM), osteoporosis, hypertension, infections and psychosis. Most of these adverse effects are usually related to high doses and/or prolonged treatment and resolve after discontinuation of steroid treatment. Nevertheless, it seems that the administration of corticosteroids in DILI has not been reported as detrimental in these patients.

Mild adverse effects of corticosteroid treatment in patients with DILI have though been reported in some studies (Hu et al., 2016). Among 53 patients receiving corticosteroids for DILI, three patients developed respiratory infections (successfully treated with antibiotics), two patients with DM had uncontrolled increase of blood glucose, one experienced mental disturbances, and another one hypertension. Since steroids improved survival in that particular study and was associated with improvement in the liver injury, the authors strongly recommended short-term administration of steroids in severe DILI patients (Hu et al., 2016).

Safety of corticosteroid use for severe DILI was also investigated in a retrospective observational study enrolling 90 patients with severe DILI (Wan et al., 2019). Among them, 66 patients were receiving a median dose of $40 \mathrm{mg}$ prednisone per day, while 24 patients composed the control group. The only adverse effects recorded were infections that occurred in 12 out of $66(18 \%)$ patients, contrary to three out of $24(12.5 \%)$ patients in the control group. However, Faje et al. (2018) reported that "high-dose" glucocorticoids (>7.5 mg prednisone or equivalent per day) for the treatment of ipilimumab-induced hypophysitis was associated with reduced survival in patients with melanoma. It was the first study reporting a potentially negative impact of corticosteroids on the efficacy of CPI treatment. In a large cohort study involving 740 melanoma patients receiving CPIs, Del Castillo et al. (2016) found that concomitant use of corticosteroids was associated with significantly higher risk of serious infections (odds ratio [OR], 7.71; 95\% confidence interval [CI], 3.71-16.18; $p<0.0001)$.

In summary, corticosteroid administration in patients with DILI is usually well tolerated, although this has not been systematically reported. Some adverse effects of corticosteroids are worrisome, particularly if higher steroid doses are used, and clinicians should carefully evaluate each case for benefits and risks.

\section{CONCLUSION}

Corticosteroids are often used in the treatment of DILI. However, their efficacy and safety are still disputable. Based on the available knowledge, patients with severe DILI or DI-AIH might benefit from steroid therapy, although evidence based on randomized controlled trials is largely lacking.

The global increase of CPI-based cancer treatment has led to a increase of indirect DILI. Hepatotoxicity occurs in $2-18 \%$ of patients treated with CPIs. Steroid therapy is not evidence based and its impact on patient outcomes is not clear. Therefore, indication, dose and duration of steroid therapy should be investigated in randomized controlled trials to fill the lack of evidence in the current treatment guidelines regarding efficacy, survival and side effects. 


\section{DATA AVAILABILITY STATEMENT}

The original contributions presented in the study are included in the article/Supplementary Material, further inquiries can be directed to the corresponding author.

\section{AUTHOR CONTRIBUTIONS}

EB designed the review and wrote the first draft. EB, VV, GS and MR-D performed the literature analysis, and took part in writing

\section{REFERENCES}

Bernal, W., Ma, Y., Smith, H. M., Portmann, B., Wendon, J., and Vergani, D. (2007). The Significance of Autoantibodies and Immunoglobulins in Acute Liver Failure: a Cohort Study. J. Hepatol. 47 (5), 664-670. doi:10.1016/j.jhep. 2007.05.011

Björnsson, E., Jerlstad, P., Bergqvist, A., and Olsson, R. (2005). Fulminant DrugInduced Hepatic Failure Leading to Death or Liver Transplantation in Sweden. Scand. J. Gastroenterol. 40 (9), 1095-1101. doi:10.1080/00365520510023846

Björnsson, E., Talwalkar, J., Treeprasertsuk, S., Kamath, P. S., Takahashi, N., Sanderson, S., et al. (2010). Drug-Induced Autoimmune Hepatitis: Clinical Characteristics and Prognosis. Hepatology 51 (6), 2040-2048. doi:10.1002/hep. 23588

Björnsson, E. S., Gunnarsson, B. I., Gröndal, G., Jonasson, J. G., Einarsdottir, R., Ludviksson, B. R., et al. (2015). Risk of Drug-Induced Liver Injury from Tumor Necrosis Factor Antagonists. Clin. Gastroenterol. Hepatol. 13 (3), 602-608. doi:10.1016/j.cgh.2014.07.062

Björnsson, E. S., Bergmann, O., Jonasson, J. G., Grondal, G., Gudbjornsson, B., and Olafsson, S. (2017). Drug-induced Autoimmune Hepatitis: Response to Corticosteroids and Lack of Relapse after Cessation of Steroids. Clin. Gastroenterol. Hepatol. 15 (10), 1635-1636. doi:10.1016/j.cgh.2017.05.027

Björnsson, H. K., Gudbjörnsson, B., and Björnsson, E. S. (2022). Infliximabinduced Liver Injury: Clinical Phenotypes, Autoimmunity and the Role of Corticosteroid Treatment. J. Hepatol. 76 (21), 86-92. Online ahead of print. doi:10.1016/j.jhep.2021.08.024

Borlak, J., van Bömmel, F., and Berg, T. (2018). N-acetylcysteine and Prednisolone Treatment Improved Serum Biochemistries in Suspected Flupirtine Cases of Severe Idiosyncratic Liver Injury. Liver Int. 38, 365-376. doi:10.1111/liv.13538

Brahmer, J. R., Lacchetti, C., Schneider, B. J., Atkins, M. B., Brassil, K. J., Caterino, J. M., et al. (2018). Management of Immune-Related Adverse Events in Patients Treated with Immune Checkpoint Inhibitor Therapy: American Society of Clinical Oncology Clinical Practice Guideline. J. Clin. Oncol. 36 (17), 1714-1768. doi:10.1200/JCO.2017.77.6385

Chalasani, N., Fontana, R. J., Bonkovsky, H. L., Watkins, P. B., Davern, T., Serrano, J., et al. (2008). Causes, Clinical Features, and Outcomes from a Prospective Study of Drug-Induced Liver Injury in the United States. Gastroenterology 135, 1924-4. doi:10.1053/j.gastro.2008.09.011

Cheung, V., Gupta, T., Payne, M., Middleton, M. R., Collier, J. D., Simmons, A., et al. (2019). Immunotherapy-related Hepatitis: Real-World Experience from a Tertiary centre. Frontline Gastroenterol. 10 (4), 364-371. doi:10.1136/flgastro2018-101146

De Martin, E., Michot, J. M., Papouin, B., Champiat, S., Mateus, C., Lambotte, O., et al. (2018). Characterization of Liver Injury Induced by Cancer Immunotherapy Using Immune Checkpoint Inhibitors. J. Hepatol. 68, 1181-1190. doi:10.1016/j.jhep.2018.01.033

Del Castillo, M., Romero, F. A., Argüello, E., Kyi, C., Postow, M. A., and RedelmanSidi, G. (2016). The Spectrum of Serious Infections Among Patients Receiving Immune Checkpoint Blockade for the Treatment of Melanoma. Clin. Infect. Dis. 63, 1490-1493. doi:10.1093/cid/ciw539

European Association for the Study of the Liver (1979). Randomised Trial of Steroid Therapy in Acute Liver Failure. Report from the European Association for the Study of the Liver (EASL). Gut 20, 620-623. doi:10.1136/gut.20.7.620 of the manuscript. All authors approved the final version of the manuscript for publication.

\section{FUNDING}

This article is based upon work from COST Action "CA17112 Prospective European DrugInduced Liver Injury Network" supported by COST (European Cooperation in Science and Technology). www.cost.eu EB, VV, GS, and MR-D are members of COST Action CA17112.

European Association for the Study of the Liver (2015). EASL Clinical Practice Guidelines: Autoimmune Hepatitis. J. Hepatol. 63, 971-1004. doi:10.1016/j. jhep.2015.06.030

Faje, A. T., Lawrence, D., Flaherty, K., Freedman, C., Fadden, R., Rubin, K., et al. (2018). High-Dose Glucocorticoids for the Treatment of Ipilimumab-Induced Hypophysitis Is Associated with Reduced Survival in Patients with Melanoma. Cancer 124 (18), 3706-3714. doi:10.1002/cncr.31629

Ferrero, D., Pogliani, E. M., Rege-Cambrin, G., Fava, C., Mattioli, G., Dellacasa, C., et al. (2006). Corticosteroids Can Reverse Severe Imatinib-Induced Hepatotoxicity. Haematologica 91 (6 Suppl. 1), ECR27. doi:10.3324/\%25x

Gauci, M. L., Baroudjian, B., Zeboulon, C., Pages, C., Poté, N., Roux, O., et al. (2018). Immune-related Hepatitis with Immunotherapy: Are Corticosteroids Always Needed? J. Hepatol. 69, 548-550. doi:10.1016/j.jhep.2018.03.034

Gauci, M. L., Baroudjian, B., Bédérède, U., Zeboulon, C., Delyon, J., Allayous, C., et al. (2021). Severe Immune-Related Hepatitis Induced by Immune Checkpoint Inhibitors: Clinical Features and Management Proposal. Clin. Res. Hepatol. Gastroenterol. 45 (2), 101491. doi:10.1016/j.clinre.2020. 06.016

Germani, G., Battistella, S., Ulinici, D., Zanetto, A., Shalaby, A., Pellone, M., et al. (2021). Drug Induced Liver Injury: from Pathogenesis to Liver Transplantation. Minerva Gastroenterol. 67 (1), 50-64. doi:10.23736/ s2724-5985.20.02795-6

Ghabril, M., Bonkovsky, H. L., Kum, C., Davern, T., Hayashi, P. H., Kleiner, D. E., et al. (2013). Liver Injury from Tumor Necrosis Factor- $\alpha$ Antagonists: Analysis of Thirty-Four Cases. Clin. Gastroenterol. Hepatol. 11, 558-e3. doi:10.1016/j. cgh.2012.12.025

Haanen, J. B. A. G., Carbonnel, F., Robert, C., Kerr, K. M., Peters, S., Larkin, J., et al. (2018). Management of Toxicities from Immunotherapy: ESMO Clinical Practice Guidelines for Diagnosis, Treatment and Follow-Up. Ann. Oncol. 29, iv264-iv266. doi:10.1093/annonc/mdy162

Hoofnagle, J. H., and Björnsson, E. S. (2019). Drug-Induced Liver Injury - Types and Phenotypes. N. Engl. J. Med. 381, 264-273. doi:10.1056/NEJMra1816149

Hou, F. Q., Zeng, Z., and Wang, G. Q. (2012). Hospital Admissions for DrugInduced Liver Injury: Clinical Features, Therapy, and Outcomes. Cell. Biochem. Biophys. 64, 77-83. doi:10.1007/s12013-012-9373-y

Hu, P. F., Wang, P. Q., Chen, H., Hu, X. F., Xie, Q. P., Shi, J., et al. (2016). Beneficial Effect of Corticosteroids for Patients with Severe Drug-Induced Liver Injury. J. Dig. Dis. 17, 618-627. doi:10.1111/1751-2980.12383

Huffman, B. M., Kottschade, L. A., Kamath, P. S., and Markovic, S. N. (2018). Hepatotoxicity after Immune Checkpoint Inhibitor Therapy in Melanoma: Natural Progression and Management. Am. J. Clin. Oncol. 41, 760-765. doi:10. 1097/COC.0000000000000374

Ito, T., Ishigami, M., Yamamoto, T., Mizuno, K., Yamamoto, K., Imai, N., et al. (2021). Clinical Course of Liver Injury Induced by Immune Checkpoint Inhibitors in Patients with Advanced Malignancies. Hepatol. Int. 15 (5), 1278-1287. doi:10.1007/s12072-021-10238-y

Karkhanis, J., Verna, E. C., Chang, M. S., Stravitz, R. T., Schilsky, M., Lee, W. M., et al. (2014). Steroid Use in Acute Liver Failure. Hepatology 59 (2), 612-621. doi:10.1002/hep. 26678

Kirk, A. P., Jain, S., Pocock, S., Thomas, H. C., and Sherlock, S. (1980). Late Results of the Royal Free Hospital Prospective Controlled Trial of Prednisolone Therapy in Hepatitis B Surface Antigen Negative Chronic Active Hepatitis. Gut 21 (1), 78-83. doi:10.1136/gut.21.1.78 
Kitagataya, T., Suda, G., Nagashima, K., Katsurada, T., Yamamoto, K., Kimura, M., et al. (2020). Prevalence, Clinical Course, and Predictive Factors of Immune Checkpoint Inhibitor Monotherapy-Associated Hepatitis in Japan. J. Gastroenterol. Hepatol. 35 (10), 1782-1788. doi:10.1111/jgh.15041

Miller, E. D., Abu-Sbeih, H., Styskel, B., Nogueras Gonzalez, G. M., Blechacz, B., Naing, A., et al. (2020). Clinical Characteristics and Adverse Impact of Hepatotoxicity Due to Immune Checkpoint Inhibitors. Am. J. Gastroenterol. 115 (2), 251-261. doi:10.14309/ajg.0000000000000398

Ostapowicz, G., Fontana, R. J., Schiødt, F. V., Larson, A., Davern, T. J., Han, S. H., et al. (2002). Results of a Prospective Study of Acute Liver Failure at 17 Tertiary Care Centers in the United States. Ann. Intern. Med. 137 (12), 947-954. doi:10. 7326/0003-4819-137-12-200212170-00007

Pang, L., Yang, W., and Hou, F. (2018). Features and Outcomes from a Retrospective Study of 570 Hospitalized Chinese Patients with DrugInduced Liver Injury. Clin. Res. Hepatol. Gastroenterol. 42, 48-56. doi:10. 1016/j.clinre.2017.08.003

Parlati, L., Vallet-Pichard, A., Batista, R., Hernvann, A., Sogni, P., Pol, S., et al. (2018). Incidence of Grade 3-4 Liver Injury under Immune Checkpoints Inhibitors: a Retrospective Study. J. Hepatol. 69 (6), 1396-1397. doi:10.1016/ j.jhep.2018.08.014

Pollack, M. H., Betof, A., Dearden, H., Rapazzo, K., Valentine, I., Brohl, A. S., et al. (2018). Safety of Resuming Anti-PD-1 in Patients with Immune-Related Adverse Events (irAEs) during Combined Anti-CTLA-4 and Anti-PD1 in Metastatic Melanoma. Ann. Oncol. 29, 250-255. doi:10.1093/annonc/ mdx642

Puzanov, I., Diab, A., Abdallah, K., Bingham, C. O., Brogdon, C., Dadu, R., et al. (2017). Managing Toxicities Associated with Immune Checkpoint Inhibitors: Consensus Recommendations from the Society for Immunotherapy of Cancer (SITC) Toxicity Management Working Group. J. Immunother. Cancer 5 (1), 95-28. doi:10.1186/s40425-017-0300-z

Rakela, J., Mosley, J. W., Edwards, V. M., Govindarajan, S., and Alpert, E. (1991). A Double-Blinded, Randomized Trial of Hydrocortisone in Acute Hepatic Failure. The Acute Hepatic Failure Study Group. Dig. Dis. Sci. 36 (9), 1223-1228. doi:10.1007/BF01307513

Reuben, A., Koch, D. G., and Lee, W. M. (2010). Drug-Induced Acute Liver Failure: Results of a U.S. Multicenter, Prospective Study. Hepatology 52 (6), 2065-2076. doi:10.1002/hep.23937

Riveiro-Barciela, M., Barreira-Díaz, A., Vidal-González, J., Muñoz-Couselo, E., Martínez-Valle, F., Viladomiu, L., et al. (2020). Immune-related Hepatitis Related to Checkpoint Inhibitors: Clinical and Prognostic Factors. Liver Int. 40, 1906-1916. doi:10.1111/liv.14489

Rodrigues, S., Lopes, S., Magro, F., Cardoso, H., Horta e Vale, A. M., Marques, M., et al. (2015). Autoimmune Hepatitis and Anti-tumor Necrosis Factor Alpha Therapy: A Single center Report of 8 Cases. World J. Gastroenterol. 21, 7584-7588. doi:10.3748/wjg.v21.i24.7584
Shah, P., Sundaram, V., and Björnsson, E. (2020). Biologic and Checkpoint Inhibitor-Induced Liver Injury: A Systematic Literature Review. Hepatol. Commun. 4, 172-184. doi:10.1002/hep4.1465

Stravitz, R. T., Lefkowitch, J. H., Fontana, R. J., Gershwin, M. E., Leung, P. S., Sterling, R. K., et al. (2011). Autoimmune Acute Liver Failure: Proposed Clinical and Histological Criteria. Hepatology 53 (2), 517-526. doi:10.1002/hep.24080

Suzuki, A., Brunt, E. M., Kleiner, D. E., Miquel, R., Smyrk, T. C., Andrade, R. J., et al. (2011). The Use of Liver Biopsy Evaluation in Discrimination of Idiopathic Autoimmune Hepatitis versus Drug-Induced Liver Injury. Hepatology 54 (3), 931-939. doi:10.1002/hep.24481

Wan, Y. M., Wu, J. F., Li, Y. H., Wu, H. M., Wu, X. N., and Xu, Y. (2019) Prednisone Is Not Beneficial for the Treatment of Severe Drug-Induced Liver Injury: an Observational Study (STROBE Compliant). Medicine (Baltimore) 98, e15886. doi:10.1097/MD.0000000000015886

Ware, A. J., Jones, R. E., Shorey, J. W., and Combes, B. (1974). A Controlled Trial of Steroid Therapy in Massive Hepatic Necrosis. Am. J. Gastroenterol. 62, 130-133.

Weber, S., Benesic, A., Rotter, I., and Gerbes, A. L. (2019). Early ALT Response to Corticosteroid Treatment Distinguishes Idiosyncratic Drug-Induced Liver Injury from Autoimmune Hepatitis. Liver Int. 39, 1906-1917. doi:10.1111/ liv.14195

Wei, G., Bergquist, A., Broomé, U., Lindgren, S., Wallerstedt, S., Almer, S., et al. (2007). Acute Liver Failure in Sweden: Etiology and Outcome. J. Intern. Med. 262 (3), 393-401. doi:10.1111/j.1365-2796.2007.01818.x

Wree, A., Dechêne, K., Herzer, W. K., Hilgard, A., Syn, W. K., Gerken, G., et al. (2011). Steroid and Ursodesoxycholic Acid Combination Therapy in Severe Drug-Induced Liver Injury. Digestion 84, 54-59. doi:10.1159/000322298

Conflict of Interest: The authors declare that the research was conducted in the absence of any commercial or financial relationships that could be construed as a potential conflict of interest.

Publisher's Note: All claims expressed in this article are solely those of the authors and do not necessarily represent those of their affiliated organizations, or those of the publisher, the editors and the reviewers. Any product that may be evaluated in this article, or claim that may be made by its manufacturer, is not guaranteed or endorsed by the publisher.

Copyright (c) 2022 Björnsson, Vucic, Stirnimann and Robles-Díaz. This is an openaccess article distributed under the terms of the Creative Commons Attribution License (CC BY). The use, distribution or reproduction in other forums is permitted, provided the original author(s) and the copyright owner(s) are credited and that the original publication in this journal is cited, in accordance with accepted academic practice. No use, distribution or reproduction is permitted which does not comply with these terms. 